\title{
Supporting tools to technical advance in the early stages of design
}

\author{
Abderrahmen Aridhi ${ }^{1,}{ }^{*}$, Jose Jorge Espi ${ }^{2}$, Juan Pedro Berro ${ }^{3}$, and Mireia Mesas ${ }^{2}$ \\ ${ }^{1}$ Altair Engineering France, 92184 Antony Cedex, France \\ ${ }^{2}$ EURECAT, Centre Tecnològic de Catalunya, Plaça de la Ciència, 2, 08242, Barcelona, Spain \\ ${ }^{3}$ EDP Sciences, Production Department, 91944 Les Ulis Cedex A, France
}

\begin{abstract}
The development of methods and tools to support the advancement in scientific and technical solutions has emerged in recent years in order to assist decisions that allow to avoid extra efforts due to the common traditional "trial and error" approach. At the same time, new challenges in terms of environmental protection have also engaged to adopt decisions having into account that climate protection needs to remain as a primer driver in the development of the aviation sector. AIRPOXY will recover all current requirements through an integrated approach where LCA (Life Cycle Assessment), LCC (Life Cycle Cost analysis), HHRA (Human Health Risk Assessment) and numerical simulation of manufacturing processes will work together in order to demonstrate and support the development of thermoformable, repairable and bondable smart epoxy based composites for aero structures. By considering all stated before, the final aim will be double. On one hand, to be informed about technical, environmental, economic and safety requirements during key stages, in order to take informed decisions and optimise it following the Eco-design principles. On the other hand, to obtain objective data to support performance in order to increase the impact of the project and support the further implementation of the technologies as the AIRPOXY solutions reach higher TRLs.
\end{abstract}

\section{Introduction}

The Roadmap to a Resource Efficient Europe [1], aims to develop new products and processes which contribute to long term sustainability and consequently, a reduced environmental impact. Early stages of design are key to meet companies and sector challenges, especially in aeronautic industry. In that sense, a multitude of design methodologies have been developed and been used frequently in search of technologies and products that combine technical and environmental requirements, raising the added value of the product and satisfying the requirements of aerospace sector into more sustainable products. Demands of aeronautics concerning its environmental performance are established via the Advisory Council for Aviation Research and Innovation in Europe (ACARE) which has established the named "Flightpath 2050 Goals" [2]. These objectives state a $75 \%$ reduction in $\mathrm{CO} 2$ emissions, a $90 \%$ reduction in NOx emissions and a perceived noise from aircraft by $65 \%$ relative to a 2000 baseline. And this must be aligned with a whole strategy that, especially in aeronautics, has being more addressed to develop solutions according to a "Just in time" perspective, through a Total Quality Management (TQM) approach leaded by a continuing expansion in demand. Consequently, final aim must be to reduce material

\footnotetext{
*Corresponding authors: aaridhi@altair.com; josejorge.espi@eurecat.org
} 
requirements, maximize energy efficiency and as a whole, an efficient resource use. For that, innovative strategies have to be considered in the early stages of design.

Efforts on it are very frequent especially from an environmental and economic point of view. In that sense, it has long been known that the inclusion of the environmental factor at the design stage offers better results in minimizing the environmental impacts associated with a product or process. This fact has been indicated by various authors $[3,4,5]$, mentioning that majority of product costs and environmental impacts during their manufacturing, use and disposal, are mainly determined during the design phase. But not only the design affects the environmental aspect of the product but also the functional and cost requirements. A design capable of simultaneously reducing the environmental impact and external costs also allows the manufacturer to follow a strategy of maximum benefit, being able to increase the value of the product towards the consumer [6]. According to some references, from $70 \%$ [7] to $80 \%$ [8] of the final cost of a product is determined in the design phase. This can also be extended to functional requirements and environmental impacts.

The AIRPOXY project is considering the global approach described above. Its main aim is to reduce production and maintenance, repair and operating (MRO) costs of CC parts in aeronautics by introducing a new family of enhanced thermoset composites that preserve all the advantages of conventional thermosets, while showing new unprecedented features such as Re-processability, Reparability and Recyclability. AIRPOXY is a European Consortium project which has received funding from the European Union's Horizon 2020 research and innovation programme under grant agreement No 769274.

Within the project, numerical simulation is becoming a powerful tool which can reduce design time and, moreover, time wasted on trial-error process to achieve the technical requirement and the quality required at the end of the manufacturing process. In AIRPOXY, simulation will address the process feasibility regarding material behaviour and process parameters in order to set up (in one shot) the correct process parameters. The capacity to predict the component state after process will help to optimize parameters like transfer time, tool forces, tool temperature, closure velocity and to estimate defects location. All that is performed numerically, which implies material and energy waste reduction decreasing the environmental impact of the process from the design phase.

Hence, once technical requirements considered in the design are addressed complementary with environmental and economic circumstances that obviously have a direct interrelation with social aspects, it is time to also have into consideration health and safety. The referred Flightpath 2050 accounts goals for aspects in a sector that represents the most safety mode transport. The European air transport system has less than one accident per ten million commercial aircraft flights [2]. These goals consider aspects like the unmanned air vehicles, boarding and security measures, security controls without intrusion or cyberattacks, etc. But it also put focus on hazards from environment, which has to be “...precisely evaluated and risks properly mitigated...".

This paradigm brings the necessity to overcome the well-known sustainability perspective and start to think into a "Safestainability" approach, with economic viability, environmental protection and health \& safety as key drivers, and this, with help of the proposed most reputed methodologies into the three mentioned issues respectively: Life Cycle Assessment (LCA), Life Cycle Costing (LCC) and Human Health Risk Assessment (HHRA).

AIRPOXY in that sense proposes an innovative approach combining these tools with simulation techniques and fills the gaps where more often research projects aimed to reach low TRLs. Hence, numerical simulation of manufacturing process will work together with LCA, LCC and HHRA in order to demonstrate and support the development of thermoformable, repairable and bondable smart epoxy based composites for aero structures. Through a decision support process, technicians will receive essential information which will help to accomplish project challenges and the expected "Safestainability" goals. At the same time, their application will offer objective and consolidated data to support performance in order to increase the relevance of technologies developed. 


\section{Thermoforming simulation}

Two main approaches exist in literature. In this section, we address two different aspects of thermoforming simulation: firstly, how to deal with interface adhesion, secondly how to model the fabric and resin behaviour integrating all temperature dependencies. In the ply to ply interaction a pragmatic approach will be presented using Smooth Particle Hydrodynamic (SPH) particles attached to plies to create the adhesion forces. For composite behaviour, we will present a method combining fabric material laws already available in Altair RADIOSS [9] and two different behaviours associated with resin, elastoplastic (law 73) or viscoelastic (user law) both with thermal dependency.

\subsection{Ply to ply interaction}

The first objective of this work is to integrate in Radioss a representation of the adhesion force between plies. In composite forming, the best way to capture the ply motion and defects creation is to model each ply independently. The original approach we propose is to use the SPH method to create the "adhesion" force without having an explicit link between plies. The SPH technique has been introduced by [10] and used for years to model high strains phenomena coupled with rupture [11] and fluid-solid interactions like sloshing and ditching [12]. This technique enables to get an adhesion force using the "fluid" resin behaviour provided by SPH particles. Today, resin is modelled like water fluid with increased viscosity but this will be improved during the project in order to add temperature dependency on this viscosity.

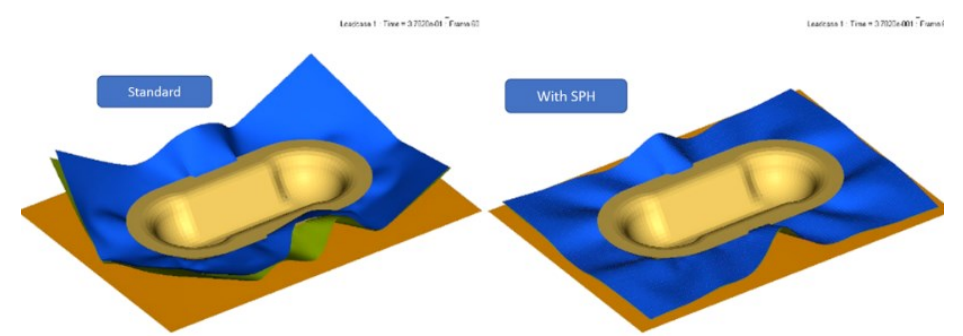

Fig. 1. Visualisation of the simulation of the process results without and without SPH particles.

\subsection{Composite material modelling}

\subsubsection{Mixed modelling}

Mixed modelling is based on the coupling of two material laws to represent the composite behaviour. We use a multilayer shell element available in Altair Radioss (/PROP/TYPE51) which allows us to combine different materials laws inside one laminate. In our case, two materials representing resin and fabric behaviour are combined. This material combination can be done in two different ways. The first option is the stacking of material plies creating a composite sandwich (resin / fabric / resin) to model one ply. The other way is to superpose two layers in the same place. Consequently, both materials will be found at the same position through the thickness. The fibre material is assumed to be hyper-elastic anisotropic. Tensile stiffness in two independent yarn directions can be defined as linear or non-linear, and softening property can be added. The shear is defined as a function of the angle between warp and weft fibre directions. As mentioned in the introduction, two different approaches to model the resin will be investigated. We need to take into account the resin influence on the global composite stiffness during the stamping process and be able to capture the strain rate 
and temperature influence on the deformation pattern. First approach is already available in RADIOSS (law 73) and consist of an elastoplastic material model based on orthotropic Hill plasticity with thermal and strain-rate dependency. In this study, we consider resin as isotropic and only thermal dependency is introduced (by curves). Yield stress is chosen very small to trigger plasticity early. In this case, we model the resin behaviour as viscoelastic adding a simplified thermal dependency. A general Maxwell element model is implemented [13] with 4 viscous contributions. Following the works of Guzman et al, we introduce a linear dependency with respect to the temperature and only two Maxwell elements are used.

\subsubsection{Hypoelastic approach}

Generally, the hypo-elastic laws are widely used in FE codes to model the composite's behaviour at large strain. The hypo-elastic behaviour does not relate the stress to the strain as the elastic constitutive, but it relates the objective derivative of the Cauchy stress tensor $\underline{\underline{\sigma}}^{\nabla}$ to the strain rate $\underline{\underline{D}}$ by a constitutive tensor $\underline{\underline{\underline{C}}}$. Hypoelastic laws have the following form:

$$
\underline{\underline{\sigma}}^{\nabla}=\underline{\underline{E}}: \underline{\underline{D}}
$$

In large deformation problem, the objective derivative of the Cauchy stress tensor is used in order to eliminate the stress variation due to rigid body rotations in $\underline{\underline{\underline{\sigma}}}=\frac{d \sigma}{d t}$. In this paper an objective derivative based on the fibre rotation tensor $\triangleq$ which is the rotation from the fibre direction in the initial orthogonal frame to the current directions. In this case, the objective derivative of the Cauchy stress tensor has the following expression:

$$
\underline{\underline{\sigma}}^{\nabla}=\underline{\underline{\Delta}}\left(\frac{d}{d t}\left(\underline{\underline{\Delta}}^{T} \underline{\underline{\sigma}} \underline{\underline{\Delta}}\right)\right) \underline{\underline{\Delta}}^{T}
$$

The first vectors $\underline{f}_{1}$ and $\underline{\mathrm{f}}_{2}$ coincide with the current directions of warp and weft. In fact, the two vectors are no unit but for simplicity they are chosen to be unit vectors. Initially, $\mathrm{f}_{1}$ and $\underline{\mathrm{f}}_{2}$ are orthogonal. Let $\underline{f}_{1}^{0}$ and $\underline{f}_{2}^{0}$ denote the fibre directions in the initial configuration. In the current configuration, the fibre directions are obtained from the deformation gradient tensor $\underline{F}$ and the initial fibre vectors [abd]. The work basis of RADIOSS is the elemental frame, defined from the natural (covariant) vectors. The elemental basis is defined by the two orthogonal vectors $\underline{t}_{1}$ and $\underline{t}_{2}$. The latter vectors are updated using the tensor $\underline{R}^{*}$ and the initial vectors $\underline{t}_{1}^{0}$ and $\underline{t}_{2}^{0}$ :

$$
\underline{t}_{i}=\underline{R}^{*} \cdot \underline{t}_{i}^{0} i=1,2
$$

Initially, the vectors of the two bases (fibre and elemental) are assumed coincided and orthogonal. But after deformation the basis $\left(\underline{f}_{1} ; f_{2}\right)$ does not remain orthogonal. Therefore, two orthogonal bases are constructed in order to track the fibre directions. The first basis denoted $B_{1}=\left(\underline{f}_{1} ; \underline{f}^{2} /\left\|\underline{f}^{2}\right\|\right)$, is based on the first fibre vector $\underline{f}_{1}$ and its contravariant vector $\underline{f}^{2}$. The second orthogonal basis $B_{2}=\left(\underline{f}^{1} /\left\|f_{\underline{f}}^{1}\right\| ; f_{2}\right)$, is defined from the second fibre vector $f_{2}$ and the first vector $f^{1}$ of the contravariant basis. $B_{e}=\left(\underline{t}_{1} ; \underline{t}_{2}\right)$ denotes the elemental basis of RADIOSS. The present model was implemented in RADIOSS via a user material subroutine in Fortran. At each increment time, Radioss calculated the strain increment $[\mathrm{d} \varepsilon]_{\mathrm{e}}$ and expressed it in the elemental frame. Therefore, the strain increment has to be converted to both orthogonal fibre bases. 

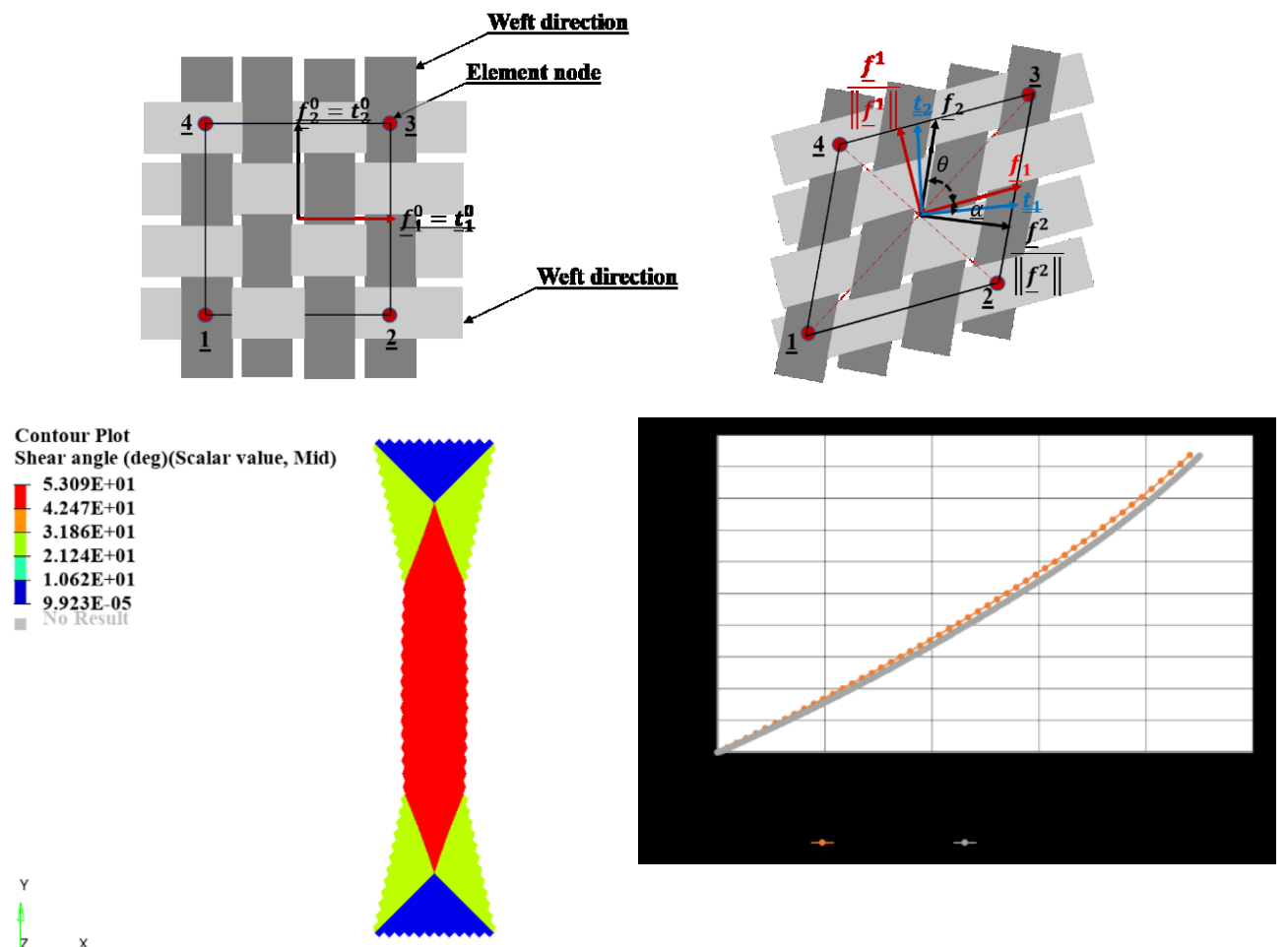

Fig. 2. Orthogonal bases, bias test specimen and bias shear angle-displacement curves.

The strain increment $\mathrm{d} \varepsilon_{11}^{\mathrm{f}_{1}}$ in the first fibre direction is obtained from the first component of $[\mathrm{d} \varepsilon]_{\mathrm{B}_{1}}$. Similarly, the strain increment $\mathrm{d} \varepsilon_{11}^{\mathrm{f}_{1}}$ in the second fibre direction is deducted from the second component of $[\mathrm{d} \varepsilon]_{\mathrm{B}_{2}}$. Whereas the in-plane shear strain increment, defined as the angle between the warp and weft yarns, is calculated by the sum of the components of shear strain increments of $[\mathrm{d} \varepsilon]_{\mathrm{B}_{1}}$ and $[\mathrm{d} \varepsilon]_{\mathrm{B}_{2}}$ as $\mathrm{d} \gamma=\mathrm{d} \varepsilon_{12}^{\mathrm{f}_{1}}+\mathrm{d} \varepsilon_{12}^{\mathrm{f}_{2}}$. In the two orthogonal fibre bases, the stress increment is related to the strain increment by the following equation:

$$
[\mathrm{d} \sigma]_{\mathrm{B}_{\mathrm{i}}}=[\mathrm{C}]_{\mathrm{B}_{\mathrm{i}}}[\mathrm{d} \varepsilon]_{\mathrm{B}_{\mathrm{i}}}
$$

Where, $[\mathrm{C}]_{\mathrm{B}_{1}}$ and $[\mathrm{C}]_{\mathrm{B}_{2}}$ are the constitutive matrices, expressed in the orthogonal fibre bases by using the tensile modulus in each fiber direction $\left(\mathrm{E}_{1}\right.$ and $\left.\mathrm{E}_{2}\right)$ and the in-plane shear modulus $G_{12}$. The stresses in the fiber frames are updated according to the following expression:

$$
[\sigma]_{\mathrm{B}_{1}}^{\mathrm{n}+1}=[\sigma]_{\mathrm{B}_{1}}^{\mathrm{n}}+[\mathrm{d} \sigma]_{\mathrm{B}_{1}}^{\mathrm{n}+1 / 2} \quad[\sigma]_{\mathrm{B}_{2}}^{\mathrm{n}+1}=[\sigma]_{\mathrm{B}_{2}}^{\mathrm{n}}+[\mathrm{d} \sigma]_{\mathrm{B}_{2}}^{\mathrm{n}+1 / 2}
$$

The total stress expressed in the elemental basis used by Radioss is obtained from the previous equations:

$$
[\sigma]_{\mathrm{B}_{\mathrm{e}}}^{\mathrm{n}+1}=[T]_{1}[\sigma]_{\mathrm{B}_{1}}^{\mathrm{n}+1}[\mathrm{~T}]_{1}^{\mathrm{T}}+[T]_{2}[\sigma]_{\mathrm{B}_{2}}^{\mathrm{n}+1}[\mathrm{~T}]_{2}^{\mathrm{T}}
$$

This model has been tested using data from a bias test. In the bias extension simulation, shell elements are used and oriented at $45^{\circ}$ regarding the load axis. A total displacement $d=$ $50 \mathrm{~mm}$ was applied to one side of the specimen. Figure 2 (centre) illustrates the distinct zones of the shear angle. The central zone (red) is the zone characterised by a pure shear state. 
However, the blue zone doesn't have any deformation. In the green zone the value of deformation is the half of that of the red zone. Figure 2 (right) shows the evolution of the numerical and the theoretical shear angle in the pure shear zone. The bias test simulation shows that the numerical results confirm the theoretical results of the shear angle evolution in the central zone.

\section{LCA, LCC and HHRA for the "Safestainability" of the solutions}

\subsection{Methodology}

AIRPOXY innovative products, since an environmental perspective, will be easily processed and repaired, and even recycled, with the added value which these properties will confer products to reduce natural sources use.

The perspective selected is cradle-to-grave LCA study. It focusses on the assessment of the benefits and drawbacks related to the environmental impact of the new developments having into account three main aspects of the life cycle: product manufacturing, use in real environment, and recycling options. Specific LCA assemblies are being modelled to assess any environmental aspect of relevance along all stages in accordance with specific objectives of the AIRPOXY project. Specific modules for each of the manufacturing stage are being constructed: production of the new 3R resin, sheet manufacturing by RTM and for thermoforming processes, bonding processes, systems for monitoring and in-situ repair method. In particular, the environmental studies performed along the project are following the recommendations set in the ILCD Handbook published by the European Commission [17] as well as the recommendations set in the 14.040 ISO standards [18]. A parametric tool developed in Excel ${ }^{\circledR}$ spreadsheet, coupled to specific LCA software is being elaborated to determine the environmental profile of any scenario analysed. This approach allows obtaining a decision support tool which helps to include environmental criteria during process development and the quantification of the environmental gain expected from the innovative alternatives. This is especially relevant in that case and allows to, as described at the beginning, identify possible improvements to goods and services in the form of lower environmental impact and lower economical cost across all life cycle stages.

With all stated, the specific goals and scope of the LCA as well as the purposes of the LCA have being defined: To be informed about the environmental impact during key stages in order to take informed decisions and optimise it following the Eco-design principles; To obtain objective data to communicate environmental performance in order to increase the impact of the project; And finally, to support the further implementation of the technologies as the AIRPOXY solutions reach higher TRLs.

To optimise and demonstrate the economic sustainability of the proposed concept, the Life Cycle Costing (LCC) methodology is being implemented in order to predict and assess the cost performance of the new AIRPOXY solutions. In particular, the LCC study is focussed on the quantification of the economic savings during manufacturing stages due to a limited generation of scrap, a lower use of energy and some minimized labour efforts, all those for the expected economic savings due to reduced inspection and repair costs. The LCC study will follow the recommendations set in the Code of Practice on LCC published by SETAC [19] mainly. It entails same scheme and modules described for LCA.

Finally, the safety of the new AIRPOXY processing technologies and resins is evaluated in terms of human health risk assessment (HHRA) considering the health effects associated to the exposure of workers to chemical compounds during the manufacturing process. The main objectives are firstly to assure their safe use and secondly to compare the new AIRPOXY processes and resins with the traditional ones with respect to their risk for the human health. HHRA is a qualitative and quantitative process that allows to determine the probability of adverse effects for people exposed to compounds and chemical elements 
gradually. During ARPOXY project, HHRA will be assessed using Standard methodology, following the Technical Guidance Document on Risk Assessment from EU [20]. Hence, the different stages considered for HHRA are the following: conceptual model, toxicity assessment, exposure assessment and risk characterization

In that sense, Life Cycle Assessment (LCA) will address eco-design criteria and end-oflife aspects to demonstrate these benefits, Life Cycle Cost (LCC) will help in selecting the most suitable options and finally, Human Health Risk Assessment (HHRA) will assess risk of the new processing technologies and the new resins accordingly. Hence, it has to be demonstrated that these new resins will:

- Reduce fuel consumption and $\mathrm{CO}_{2}$ emissions of airplanes where parts will be manufactured with the proposed technologies thanks to weight reduction

- Reduce the quantity of landfilled waste by demonstrating benefits from the recyclability and reuse enhanced properties of AIRPOXY products

- Reduce manufacturing costs of composite parts in 37\% compared with autoclave processes

- Reduce assembly costs of composite parts in 53\% compared with adhesive bonding, repairing costs in 50\% compared with patching process and reduce in $90 \%$ the labour costs

Two representative demonstrators of typical aero structures will be manufactured to validate the technologies: a sub-component of a fan-cowl formed by a longitudinal stiffener, transvers stiffener and skin, and a sub-component of a leading-edge formed by a nose and two webs, that will be the basis of the studies. By the analysis of these structures manufactured with newer technologies and comparing them against conventional technologies, all these improvements will be demonstrated.

\section{Work so far}

Currently, goal \& scope definition of the proposed solutions being developed have been described along the present paper. Hence, project is being dedicated to the inventory stage, where it has been identified around 100 different processes and sub-process along life cycle of the demonstrators. With the help of all AIRPOXY consortia members, questionnaires to reflect the different scenarios and for data gathering has been released and partners have started to acquire data from previous knowledge and tests performed. First efforts are conducted to construct the Life Cycle Model as well as the scenarios and receptors definition for the HHRA studies. In that sense, numerical simulations are paying a special role. Thanks to the use of relevant information concerning the properties of materials used, the design implications proposed and tested as the relevant information for inventory gathering like energy consumption, amounts of material, and expected lifetime of structures, LCA, LCC and HHRA are being providing of information and validating any proposal through the mentioned "safestainaibility" perspective. Hence, the decision process mechanism is aligned in any time with project objectives and results concerning the performance of the demonstrators will be in that sense well-described and validated.

\section{Conclusions}

Some numerical tools have been developed in order to better represent the $3 \mathrm{R}$ composites thermoforming process. These developments will allow to perform accurate thermoforming simulations thanks to the better ply to ply interaction phenomenon integration on thermoforming simulations as well as the composite material behaviour temperature dependency. The better accuracy allows to predict defects on the final component and to optimize the process parameters (in terms of quality, energy, time...). Other benefit of the process simulation is the ability of quantifying the material and energy consumption, to use in LCA, LCC analysis. 
Info generated will serve to support technical as well as environmental, economic and safety studies especially those with high uncertainty to be recovered by primary data. Aspects like the weight reduction, cheaper and better recyclability of materials, energy efficiency, as well as reduced repairing and maintenance operations will assure successful of the solutions proposed. Thanks to that, any technical decision will be open to consider environmental, economic, and safety circumstances and a process to improve hotspots will be addressed since the beginning, in a continuous monitoring way and will assure "Safestainability" of the solutions provided.

\section{References}

1. Communication from the commission to the European parliament, the council, the European economic and social committee and the committee of the regions. Roadmap to a Resource Efficient Europe. (2011) COM/2011/0571 final

2. Acare4europe.org. (2019). FlightPath 2050 Goals | Acare. [online] Available at: https://www.acare4europe.org/sria/flightpath-2050-goals [Accessed 22 Sep. 2019].

3. A. Chaabane, A. Ramudhin, M. INT. J. PROD. ECON. 135, 37 (2012).

4. D. Maxwell, R. Van der Vorst. J. CLEAN. PROD. 11, 883 (2003).

5. R. Nidumolu, C.K. Prahalad, M.R. Rangaswami. HARV. BUS. REV. 87, 56 (2009).

6. J. Jeswiet, M. Hauschild. MATER DESIGN, 26, 629 (2005).

7. G. Boothroyd. COMPUT. AIDED. DESIGN. 26, 505 (1994).

8. S. Ferrendier, F. Mathieux, G. Rebitzer, M. Simon, D. Froelich. Eco-Design GuideEnvironmentally Improved Product Design Case Studies of the European Electrical and Electronic Industry. EU Ecolife Thematic Network (2002).

9. RADIOSS Users Manual, Version 2019

10. J. J. Monaghan. COMPUT. PHYS. COMMUN. 48 , 89 (1988).

11. M. B. Liu, G. R. Liu, K. Y. Lam. SHOCK WAVES 15, 21 (2006).

12. M. Siemann, P. Groenenboom. Modelling and validation of guided ditching tests using a coupled SPH-FE approach. In: Proceedings of 9th SPHERIC international workshop. (2014). p. 260-268 (2014).

13. W. K. Liu. Finite element procedures for fluid-structure interactions and application to liquid storage tanks. NUCL. ENG. DES. 65, 221 (1981).

14. EU-European Commission. "International reference life cycle data system (ILCD) handbook - General guide for life cycle assessment-Detailed guidance." Institute for Environment and Sustainability (2010).

15. ISO 14040: Environmental Management - Life Cycle Assessment. - Principles and Framework

16. T. E. Swarr, D. Hunkeler, W. Klöpffer, H. L. Pesonen, A. Ciroth, A. C. Brent, R. Pagan. Environmental life-cycle costing: a code of practice (2011).

17. European Commission. "Technical Guidance Document on Risk Assessment in support of Commission Directive 93/67/EEC" (2003). 